\title{
El funcionamiento del Consejo Técnico, sus orientaciones y su tipología
}

\author{
Cruz Argelia Estrada Loya \\ Centro Chihuahuense de Estudios de Posgrado \\ dreduarely@hotmail.com
}

\begin{abstract}
Resumen
El Consejo Técnico es un órgano legalmente establecido en las escuelas primarias mexicanas que instituye el trabajo colaborativo de la planta docente para la mejora académica. El presente trabajo muestra los resultados de un trabajo de investigación realizado en escuelas primarias de la Región Centro en el estado de Chihuahua en el cual se describen los rasgos característicos, organizados en ocho categorías, que muestran el funcionamiento de dicho órgano y las orientaciones que frecuentemente tienen las actividades que de él emergen, mismas que conllevan a la identificación de cuatro tipos diferentes de organizarlo, integrarlo, percibirlo y trabajarlo.
\end{abstract}

\section{Palabras clave}

Educación básica, cultura organizacional, trabajo en equipo.

\section{Problema de estudio}

El trabajo colaborativo representa la oportunidad de minimizar esfuerzos y optimizar resultados utilizando el mínimo de recursos; en las escuelas primarias de México este tipo de trabajo se encuentra instituido en el Consejo Técnico Escolar, el cual es un órgano consultivo creado y normado en cumplimiento al Acuerdo 96 que establece la organización y funcionamiento de las escuelas primarias a nivel nacional. Dicho órgano tiene como propósito el intercambio y análisis de la tarea docente dentro del contexto específico en que ésta se desarrolla (Fierro y Rojo, 1994).

El Consejo Técnico permite que la solución de problemas y la organización de cada institución emerja de dentro hacía fuera con la propuesta de soluciones basadas en los recursos con que se cuenta y diseñadas de acuerdo a las necesidades sentidas en cada colectivo.
Dada la diversidad de actores que conforman cada comunidad educativa, así como la convergencia de diversas culturas al interior de las mismas, cada escuela presenta una realidad única e irrepetible. De aquí se deriva que a pesar de que el Consejo Técnico sea un espacio instituido normativamente en todas las escuelas primarias y reconocido como una oportunidad para la mejora educativa, no exista un funcionamiento claramente definido y establecido; el estado de conocimiento da cuenta de que el trabajo colegiado no es una realidad única y exitosa.

En consideración a dichos factores, la pregunta central que orientó esta investigación es: ¿Cómo funciona el Consejo Técnico en las escuelas primarias estatales de la Región Centro en el Estado de Chihuahua?

El trabajo se realizó con el objetivo general de describir el funcionamiento del 
Consejo Técnico en las escuelas del estudio para definir una tipología generalizada y encontrar la existencia o no de una relación entre esa tipología y la eficacia escolar.

Para alcanzar dicho objetivo, se consideraron cuatro conceptos clave, que orientaron el análisis y la discusión de los hallazgos, éstos son: calidad educativa, escuelas eficaces, trabajo colaborativo y Consejo Técnico.

Calidad educativa se define como una cualidad que caracteriza a la unidad educativa por su mejora continua; se entiende como un proceso cíclico en espiral ascendente, que es dinámico y relativo. Incluye por lo menos cinco condiciones interrelacionadas e interdependientes: relevancia, eficacia, eficiencia, equidad y pertinencia (Schmelkes, 1992). Es una cualidad distintiva que tiene el reconocimiento de "marca de calidad" por la comunidad en que se encuentra inserta (Martínez, 2003). La eficacia escolar es una de las condiciones para que haya calidad, es la forma en que una institución educativa alcanza los objetivos planteados y la cobertura de los mismos. Ésta, sienta las bases para definir las escuelas eficaces, mismas que se consideran tales si promueven el progreso para todos sus alumnos y alumnas en forma equitativa y garantizan que cada uno alcance el máximo posible, siendo éste siempre mayor a las expectativas dadas por los factores contextuales (Loera, 2006).

Se considera trabajo colaborativo a la capacidad de un grupo para trabajar de forma interdependiente con la intención de conseguir un objetivo común; el éxito de un colectivo involucra la cooperación que sus integrantes tengan en el trabajo. Las relaciones que se establezcan en el grupo son importantes tanto como mantener presente que el propósito primordial de estar reunidos es el cumplimiento de una tarea compartida. El trabajo grupal caracterizado por la división del trabajo y el reparto de fragmentos entre los integrantes del grupo, con la consecuente suma de partes para presentar como un todo el trabajo final, no corresponde a la definición de trabajo colaborativo.

El Consejo Técnico, es un órgano interno de las escuelas cuya existencia pretende la discusión y el intercambio académico de los docentes; es “...un órgano integrado por toda la planta docente o su representación, presidido por el director y encaminado a apoyar su labor, gracias a la consulta sobre distintos temas relacionados con la enseñanza que ahí se realiza." (Fierro y Rojo, 1994, pág.11).

\section{Metodología}

El presente trabajo se basó en una postura pragmatista al considerar que el problema y sus circunstancias dictan el tipo de método a utilizar y que por tanto es válido recolectar, analizar y vincular datos cualitativos y cualitativos para responder al planteamiento del problema (Hernández, Fernández y Baptista, 2006). Dada la naturaleza del objeto de estudio y el objetivo general que pretendía, se construyó un diseño de investigación dentro del enfoque mixto de investigación con la idea de complementariedad, de obtener dos imágenes (una cuantitativa y otra cualitativa) para tener un acercamiento a la realidad desde dos perspectivas diferentes y hacer un análisis más completo.

Se organizó el estudio en dos fases, el presente documento da cuenta únicamente de los hallazgos de la primera de ellas, por tanto sólo se describe ésta. La primera fase se integró por un diseño 
transeccional descriptivo no experimental con la idea de ubicar dentro de ocho categorías preestablecidas (función, organización, participación, tiempo, apoyos y obstáculos, definición, satisfacción y visión) el funcionamiento del Consejo Técnico en las escuelas del estudio.

En esta primera fase se aplicó la técnica de encuesta, la cual consiste en la aplicación de un mismo instrumento a los sujetos del estudio, se realizó mediante la aplicación de un cuestionario único, seccionado en dos etapas a causa de su extensión, dicho instrumento se aplicó a todas las personas que integran el Consejo Técnico de las escuelas de la muestra. Las respuestas a este cuestionario brindaron los datos para obtener una imagen descriptiva de la unidad de análisis pretendida.

Para la aplicación de los cuestionarios se conformó un equipo de encuestadores, integrado por un grupo de siete jóvenes estudiantes del Centro de Bachillerato Tecnológico Industrial (CBTI's 122) a cambio de realizar servicio social a la comunidad, los cuales fueron capacitados previo a la inmersión al campo; se les asignaron únicamente escuelas en la ciudad de Chihuahua, las escuelas foráneas fueron atendidas por la investigadora.

El cuestionario se aplicó en una muestra representativa generalizable a la región, se utilizó un proceso de muestreo cuantitativo de tipo probabilístico por muestra aleatoria simple. Para determinar la muestra se utilizó el programa de software STATSS. Se seleccionaron 48 escuelas de la región, teniendo una muestra representativa con un margen de error de 10.

Los datos obtenidos se analizaron con ayuda del programa de software SPSS.15, lo que permitió identificar dentro de cada categoría la existencia de diferentes clases para realizar la descripción de cada una de las escuelas y la elaboración de una tipología generalizada del funcionamiento del Consejo Técnico.

\section{Discusión de resultados}

Los datos obtenidos durante la primera fase del estudio brindaron tres resultados diferentes: las clases que surgieron dentro de cada categoría de estudio, las orientaciones del Consejo Técnico, y la tipología de su funcionamiento.

\section{Las categorías y sus clases}

Para describir el funcionamiento del Consejo Técnico, se diseñó un cuestionario que rescató información sobre ocho categorías organizadas en dos grupos; cinco categorías buscaron describir propiamente el funcionamiento del órgano en cuestión, mientras que las tres restantes pretendieron recoger información sobre la percepción que los encuestados tienen sobre el trabajo que desempeña. Los datos obtenidos en las preguntas realizadas se organizaron en clases dentro de cada categoría para caracterizar el comportamiento de cada una y condujeron a la descripción de cada escuela del estudio. La ilustración 1 muestra las categorías y sus clases. 


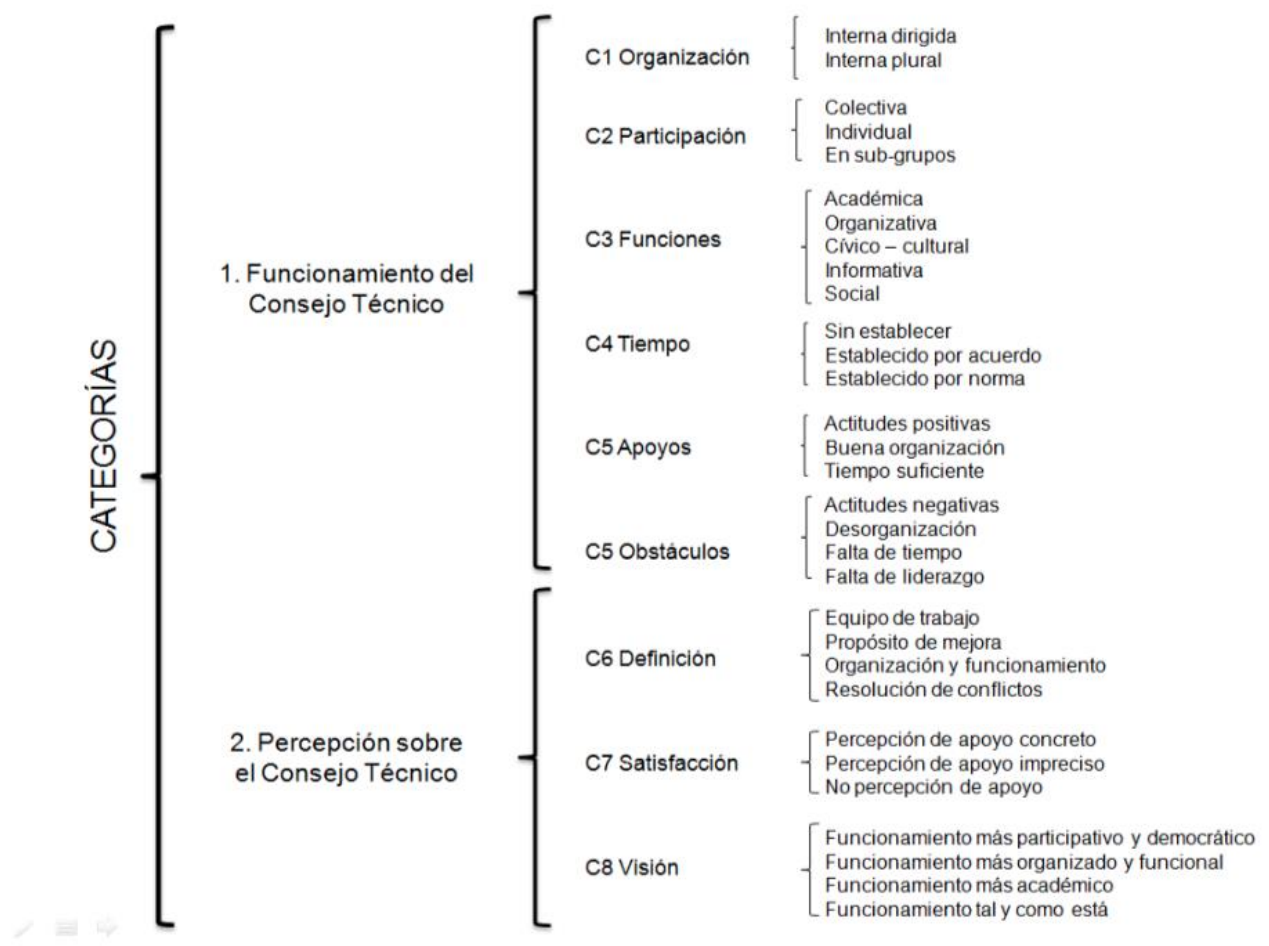

\section{Las orientaciones del Consejo Técnico}

Las respuestas que los encuestados brindaron a los cuestionamientos referidos a la categoría de funciones (C3), permitieron a la vez identificar que el Consejo Técnico, desde el marco legal, tiene un propósito eminentemente académico; sin embargo, la reglamentación para su funcionamiento así como las circunstancias y características del sistema educativo permiten que cada escuela las reinterprete de acuerdo a sus necesidades, prioridades y relaciones internas, por lo que en cada escuela se presentan diferentes orientaciones del funcionamiento del órgano en cuestión de acuerdo a las necesidades del contexto y las prioridades que el colegiado establezca.

En el proceso de investigación realizado se encontraron cinco orientaciones: la académica, la organizativa, la informativa, la cívico- cultural y la social.

Se identificó una orientación académica cuando la mayoría de las actividades que se abordan al interior del Consejo Técnico tienen que ver con la práctica educativa, sus elementos y sus resultados; se detectó una orientación organizativa cuando las acciones que se ejecutan van encaminadas a ordenar $y$ garantizar el establecimiento de condiciones básicas para el desarrollo de las actividades educativas, esto incluye el acondicionamiento y mantenimiento del edificio escolar; la orientación informativa se identificó en los espacios en los que el tiempo de reuniones de Consejo Técnico se utiliza para comunicar una serie de cuestiones administrativas que los docentes deben cumplir, frecuentemente relacionados con el llenado de papelería y la elaboración de planes e informes; la orientación cívico-cultural se manifestó cuando las actividades realizadas se 
realizan con el fin de organizar la conmemoración de fechas cívicas o bien la participación en eventos culturales convocados por la secretaría de educación; finalmente se detectó una orientación social cuando las actividades desarrolladas buscan generar y mantener un ambiente armónico en la comunidad escolar.

Las orientaciones del funcionamiento del Consejo Técnico no se presentan de forma pura, sino que generalmente se presentan dos o más de ellas, pero siempre una de ellas abarca la mayoría de las actividades que se realizan $y$ el personal les brinda mayor importancia, por lo que la mayor parte de los esfuerzos se concentran en ello.

La orientación que presenta el Consejo Técnico en las escuelas refleja de manera importante el tipo de escuela y el tipo de educación que en ella se imparte. Con el trabajo cotidiano, se gesta al interior del Consejo Técnico una cultura de funcionamiento conformada por las ideas y creencias de sus integrantes, mismas que se expresan en la forma regular $y$ frecuente de trabajarlo; esta forma de asumir y abordar el trabajo conjunto, define la orientación del mismo y constituye el núcleo de la cultura escolar.
Tipología del funcionamiento del Consejo Técnico

Los datos obtenidos mostraron que si bien no existen dos escuelas con una forma idéntica de definir, organizar, trabajar y percibir el Consejo Técnico; si comparten rasgos que conforman una cultura de funcionamiento similar que puede describirse a través de las características que lo constituyen; dicha cultura puede agruparse en cuatro tipos que, de acuerdo a las definiciones que hace Wenger (2001) de las relaciones colectivas, se definieron como: Colectivo docente, Red docente, Grupo docente y Colectivo docente. La ilustración 2 muestra en resumen la descripción de cada tipo y el porcentaje con que se presentaron en la muestra.

Los resultados mostraron que más de una tercera parte (41.4\%) de las escuelas tienen una cultura de funcionamiento por Grupo Docente y otra tercera parte $(39.1 \%)$ tiene una cultura de trabajo Colectivo Docente y menos de una tercera parte (19.5\%) se conforma por los tipos de Comunidad Docente y Red Docente, que son los más apegados al funcionamiento académico que se establece por normatividad.

Dada la selección de la muestra, estos resultados son generalizables a toda la Región Centro en el estado de Chihuahua.

\begin{tabular}{||c|c||}
\hline COMUNIDAD DOCENTE 7.3\% & RED DOCENTE 12.2\% \\
\hline Marcado esfuerzo por la mejora académica, & Esfuerzo por la mejora centrado en una \\
lo organizativo y extraescolar pasa a & buena organización, se trabaja lo académico \\
segundo término, trabajo en equipo con & como actividad inherente a la mejora, pero \\
colaboración abierta, comunicación amplia & no prioritaria; se trabaja en sub grupos que \\
y buenas relaciones interpersonales, se tiene & llegan a establecer acuerdos para eventos \\
un propósito bien definido para el trabajo y & representativos de la escuela, existe cierto \\
\hline
\end{tabular}


RECIE. Revista Electrónica Científica de Investigación Educativa Vol. 1, núm. 1, enero-diciembre 2012, pp. 121-127.

\begin{tabular}{|c|c|}
\hline se tiene disponibilidad al cambio. & $\begin{array}{l}\text { grado de satisfacción con el trabajo } \\
\text { realizado. }\end{array}$ \\
\hline GRUPO DOCENTE $41.4 \%$ & COLECTIVO DOCENTE $39.1 \%$ \\
\hline $\begin{array}{l}\text { Se da prioridad al establecimiento de las } \\
\text { condiciones básicas para la enseñanza y el } \\
\text { cumplimiento con actividades extra } \\
\text { escolares, busca la mejora académica a } \\
\text { través de capacitaciones; se trabaja en } \\
\text { pequeños sub grupos que son antagónicos, } \\
\text { las decisiones se realizan por votación y las } \\
\text { relaciones son poco democráticas. }\end{array}$ & $\begin{array}{l}\text { No se muestran esfuerzos por la mejora, se } \\
\text { centra el trabajo en lo organizativo y lo } \\
\text { cívico cultural dejando fuera el trabajo } \\
\text { académico; se presenta un trabajo individual } \\
\text { marcado por malas relaciones } \\
\text { interpersonales y un sentimiento de } \\
\text { inequidad en el cumplimiento de } \\
\text { responsabilidades; se percibe un mal } \\
\text { funcionamiento y se ubica el problema } \\
\text { fuera de la escuela. }\end{array}$ \\
\hline
\end{tabular}

Ilustración 2Tipología de funcionamiento del Consejo Técnico

El funcionamiento del Consejo Técnico es un proceso que se construye en las escuelas primarias y se ve atravesado por diversos elementos. Cada escuela tiene una forma particular de concebirlo y trabajarlo; éste se ve influenciado por la convergencia de ideologías y creencias que poseen sus integrantes ya que como lo establecen Sánchez y Ruíz (2008), la dinámica escolar se forma por la contribución personal y las relaciones o la falta de ellas entre los miembros que lo conforman, lo que explica que no existan dos escuelas con un funcionamiento idéntico del Consejo Técnico aun y cuando lleguen a compartir edificio escolar.

Puesto que dicho órgano es el encargado de analizar y recomendar en cuanto a asuntos académicos, se ha establecido para apoyar la labor de enseñanza y coadyuvar en la resolución de problemas pedagógicos por medio de la consulta y el análisis conjunto (Fierro y Rojo, 1994); se entiende que el tipo de cultura de funcionamiento configurada en su seno contribuye a las formas de concebir el aprendizaje, las formas de enseñanza aplicadas en el aula y a las formas de evaluar el aprendizaje que se presenta en las escuelas.

Tomando en consideración los hallazgos de diferentes investigaciones sobre el tema (Fierro y Rojo, 1994; Ezpeleta, 2000; Loera, 2006; Barraza y Guzmán, 2010), mismas que han establecido que el funcionamiento del Consejo Técnico se encuentra estrechamente relacionado con la cultura escolar y que el funcionamiento académico es posible aún en los contextos desfavorables, siempre y cuando, se trabaje de forma intencionada a fin de reorientar las actividades del mismo; puede concluirse que el Consejo Técnico representa el centro idóneo para promover la mejora continua.

Considerando que cada docente le aporta consciente o inconscientemente a la dinámica escolar instalada en su institución, cuando se tiene consciencia de esa aportación y ésta se orienta de forma intencionada hacía un propósito definido es posible que se obtenga el fin deseado. En la medida que los docentes sean conscientes del tipo de cultura de funcionamiento que están generando y viviendo en la escuela en que trabajan, podrán entenderse como una comunidad de práctica (Sánchez y Ruíz, 2008) que potencialmente puede obtener mejores resultados de aprendizaje si entiende la participación social como un elemento que 
tiene efectos en lo individual, en la comunidad y en la organización misma.

\section{Referencias}

Barraza, L. y Guzmán, A. (2002). El trabajo colegiado en la práctica educativa de los formadores de docentes. Revista Acoyauh, (No. 44), año XIV, abril.

Ezpeleta, J. (2000). El consejo técnico: eficacia pedagógica y estructura de poder en la escuela primaria mexicana. En: Gestión escolar (Antología), pág. 66. México: SEP.

Fierro, C. y Rojo, S. (1994). El consejo técnico, Un encuentro de maestros. México: SEP.

Hernández, R. Fernández, C. y Baptista, P. (2006) Metodología de la investigación. (4a ed.). México: Mc Graw Hill.

Loera, A. (2006). (Editor) Caja de herramientas para colectivos escolares. México: SEP.

Martínez, J. (2003). La calidad educativa, un espacio de lucha por la hegemonía. La Tarea. Revista de educación y cultura de la sección 47 del SNTE, (No. 18), diciembre. Consultada en: www.latarea.com.mx/articu/articu 18/martinez18.htm

Sammons, P. Hilman, J y Martimore, P. (1995). Características clave de las escuelas efectivas; una revisión de escuelas efectivas, investigación. Inglaterra: Instituto de Educación.

Sánchez, J. y Ruíz, A. (2008). Maestros y formación en comunidades de práctica. México: UPN.

Schmelkes, S. (1992). Hacia una mejor calidad de nuestras escuelas. México: SEP. 
RECIE. Revista Electrónica Científica de Investigación Educativa

Vol. 1, núm. 1, enero-diciembre 2012, pp. 121-127. 